\title{
A TAYLOR-DIRICHLET SERIES WITH NO SINGULARITIES ON ITS ABSCISSA OF CONVERGENCE
}

\section{E. ZIKKOS}

\begin{abstract}
G. Pólya proved that given a sequence of positive real numbers $\Lambda=\left\{\lambda_{n}\right\}_{n=1}^{\infty}$ of a density $d$ and satisfying the gap condition $\inf _{n \in \mathbb{N}}\left(\lambda_{n+1}-\lambda_{n}\right)>0$, the Dirichlet series $\sum_{n=1}^{\infty} c_{n} e^{\lambda_{n} z}$ has at least one singularity in each open interval whose length exceeds $2 \pi d$ and lies on the abscissa of convergence. This raises the question whether the same result holds for a Taylor-Dirichlet series of the form
\end{abstract}

$$
g(z)=\sum_{n=1}^{\infty}\left(\sum_{k=0}^{\mu_{n}-1} c_{n, k} z^{k}\right) e^{\lambda_{n} z}, \quad c_{n, k} \in \mathbb{C}
$$

when its associated multiplicity-sequence $\Lambda=\left\{\lambda_{n}, \mu_{n}\right\}_{n=1}^{\infty}$

$$
\left\{\lambda_{n}, \mu_{n}\right\}_{n=1}^{\infty}:=\{\underbrace{\lambda_{1}, \lambda_{1}, \ldots, \lambda_{1}}_{\mu_{1} \text {-times }}, \underbrace{\lambda_{2}, \lambda_{2}, \ldots, \lambda_{2}}_{\mu_{2} \text {-times }}, \ldots, \underbrace{\lambda_{k}, \lambda_{k}, \ldots, \lambda_{k}}_{\mu_{k} \text {-times }}, \ldots\}
$$

has the following two properties:

(1) $\Lambda$ has density $d$, that is, $\sum_{\lambda_{n} \leqslant t} \mu_{n} / t \rightarrow d$ as $t \rightarrow \infty$,

(2) $\lambda_{n}$ satisfy the gap condition $\inf _{n \in \mathbb{N}}\left(\lambda_{n+1}-\lambda_{n}\right)>0$.

In this article we present a counterexample. We prove that for any non-negative real number $d$ there exists a multiplicity-sequence $\Lambda=\left\{\lambda_{n}, \mu_{n}\right\}_{n=1}^{\infty}$ having properties (1) and (2), but with unbounded multiplicities $\mu_{n}$, such that its Krivosheev characteristic $S_{\Lambda}$ is negative. For this $\Lambda$, and based on a result obtained by O.A. Krivosheeva, we show that for any $a \in \mathbb{R}$, there exists a Taylor-Dirichlet series $g(z)$ whose abscissa of convergence is the line $\operatorname{Re} z=a$, such that $g(z)$ has no singularities on this line.

Keywords: Taylor-Dirichlet series, singularities, Fabry-Pólya

Mathematics Subject Classification: 30B50

\section{INTRODUCTION AND RESUlT}

The Fabry-Pólya gap theorem states that given a strictly increasing sequence $\Lambda:=\left\{\lambda_{n}\right\}_{n=1}^{\infty}$ of non-negative integers diverging to infinity, such that $n / \lambda_{n} \rightarrow d$ as $n \rightarrow \infty$, each power series of the form $\sum_{n=1}^{\infty} a_{n} z^{\lambda_{n}}$ with a finite radius of convergence $\rho$ has at least one singularity on each open arc whose length exceeds $2 \pi \rho d$ (see [1]). More recent related results have been obtained in [2], 3], 4].

Pólya generalized the gap result for exponential Dirichlet series as follows. Suppose that $\Lambda:=\left\{\lambda_{n}\right\}_{n=1}^{\infty}$ is a strictly increasing sequence of positive real numbers diverging to infinity having finite density $d$ and uniformly separated terms, that is,

$$
\lim _{n \rightarrow \infty} \frac{n}{\lambda_{n}}=d<\infty, \quad \text { and } \quad \inf _{n \in \mathbb{N}}\left(\lambda_{n+1}-\lambda_{n}\right)>0
$$

E. Zikkos, A Taylor-Dirichlet series With no singularities on its abscissa of CONVERGEnCE.

(C) E. Zikkos 2018.

Submitted May 30, $201 \%$. 
Consider then an exponential Dirichlet series of the form

$$
\sum_{n=1}^{\infty} c_{n} e^{\lambda_{n} z}, \quad c_{n} \in \mathbb{C},
$$

and suppose that $\tau=\lim \sup _{n \rightarrow \infty}\left(\log \left|c_{n}\right|\right) / \lambda_{n}$ is a real number. Assuming the above conditions on $\Lambda$, such a series converges pointwise and absolutely in the half-plane $H_{-\tau}:=\{z: \operatorname{Re} z<-\tau\}$ and uniformly on compact subsets of $H_{-\tau}$, thus defining an analytic function in this half-plane. The line $\operatorname{Re} z=-\tau$ is called the abscissa of convergence. Pólya proved that such a Dirichlet series has at least one singularity in each open interval whose length exceeds $2 \pi d$ and lies on the abscissa of convergence. For a proof we refer the reader to Boas [5, Thm. 12.7.8] and Levinson [6. Thm. XXIX] with similar results obtained in [7], 8]. We also point out that if the terms of the sequence are not uniformly separated, then the Pólya result does not hold. In this case it is possible that there exists a Dirichlet series with no singularities on the abscissa. A.F. Leont'ev was the first to construct such an example and more general results on analytic continuation across the abscissa were obtained by O. A. Krivosheeva [9, Thm. 3.1].

Inspired by Krivosheeva's result, our goal in this article is to prove that given any nonnegative real number $d$, there exists a Taylor-Dirichlet series of the form

$$
\sum_{n=1}^{\infty}\left(\sum_{k=0}^{\mu_{n}-1} c_{n, k} z^{k}\right) e^{\lambda_{n} z}, \quad c_{n, k} \in \mathbb{C}
$$

with no singularities on its abscissa of convergence such that its associated multiplicity-sequence $\Lambda=\left\{\lambda_{n}, \mu_{n}\right\}_{n=1}^{\infty}$ has density $d$ and positive real uniformly separated terms, that is,

$$
\lim _{t \rightarrow \infty} \frac{n_{\Lambda}(t)}{t}=d<\infty \quad \text { and } \quad \inf _{n \in \mathbb{N}}\left(\lambda_{n+1}-\lambda_{n}\right)>0 .
$$

where

$$
n_{\Lambda}(t):=\sum_{\lambda_{n} \leqslant t} \mu_{n}
$$

is the counting function of $\Lambda$. By a multiplicity-sequence $\Lambda=\left\{\lambda_{n}, \mu_{n}\right\}_{n=1}^{\infty}$ we mean a set with multiple terms

$$
\left\{\lambda_{n}, \mu_{n}\right\}_{n=1}^{\infty}=\{\underbrace{\lambda_{1}, \lambda_{1}, \ldots, \lambda_{1}}_{\mu_{1} \text {-times }}, \underbrace{\lambda_{2}, \lambda_{2}, \ldots, \lambda_{2}}_{\mu_{2} \text {-times }}, \ldots, \underbrace{\lambda_{k}, \lambda_{k}, \ldots, \lambda_{k}}_{\mu_{k} \text {-times }}, \ldots\}
$$

where $\left\{\lambda_{n}\right\}_{n=1}^{\infty}$ is a strictly increasing sequence of positive real numbers diverging to infinity, and $\left\{\mu_{n}\right\}_{n=1}^{\infty}$ is a sequence of positive integers.

We point out that the density condition implies that

$$
\lim _{n \rightarrow \infty} \frac{\log n}{\lambda_{n}}=0 \quad \text { and } \quad \lim _{n \rightarrow \infty} \frac{\mu_{n}}{\lambda_{n}}=0 .
$$

(for a proof see the Appendix). Assuming (1.4), it then follows from the results by Valiron [10] that the region of holomorphy of Taylor-Dirichlet series $(1.2)$ is the same with that of the series

$$
f^{*}(z)=\sum_{n=1}^{\infty} C_{n} e^{\lambda_{n} z}, \quad C_{n}=\max \left\{\left|c_{n, k}\right|: k=0,1,2, \ldots, \mu_{n}-1\right\} .
$$

Letting $\tau=\lim \sup _{n \rightarrow \infty} \frac{\log C_{n}}{\lambda_{n}}$, this region is the half-plane $\operatorname{Re} z<-\tau$ if $\tau \in \mathbb{R}$ and the complex plane $\mathbb{C}$ if $\tau=-\infty$. The line $\operatorname{Re} z=-\tau$ is called the abscissa of convergence of series 1.2 .

Therefore, a natural Fabry-Pólya type problem arises: if a multiplicity-sequence $\Lambda$ satisfies (1.3), does its associated Taylor-Dirichlet series (1.2) have at least one singular point in each open interval whose length exceeds $2 \pi d$ and which lies on the abscissa of convergence? 
Of course, as $\mu_{n}=1$ for all $n \in \mathbb{N}$, the answer is affirmative due to Pólya. We will show however this is not the case if the multiplicities $\mu_{n}$ are allowed to be unbounded. This is the content of Theorem 1.1. We prove our result based on the work by Krivosheeva [9]. A crucial role is played by the quantity $S_{\Lambda}$ of a multiplicity-sequence $\Lambda$, a notion introduced for the first time by Krivosheev [11]. We point out that $S_{\Lambda}$ is similar in nature with the Bernstein-Leont'ev condensation index and it measures in some sense how close the $\lambda_{n}$ 's are to each other. The definition of $S_{\Lambda}$ follows below.

Let $\Lambda=\left\{\lambda_{n}, \mu_{n}\right\}_{n=1}^{\infty}$ be a multiplicity-sequence with real or complex $\lambda_{n}$. Fix some $w \in \mathbb{C}$ and some positive real number $\eta<\frac{1}{10}$. Consider the open disk

$$
B(w, \eta|w|)=\{z:|z-w|<\eta|w|\} .
$$

For such a disk, construct the polynomial

$$
q_{\Lambda}(z, w, \eta)=\prod_{\lambda_{n} \in B(w, \eta|w|)}\left(\frac{z-\lambda_{n}}{3 \eta\left|\lambda_{n}\right|}\right)^{\mu_{n}}
$$

If $w=\lambda_{k}$ for some $k \in \mathbb{N}$, then

$$
q_{\Lambda}\left(z, \lambda_{k}, \eta\right)=\prod_{\lambda_{n} \in B\left(\lambda_{k}, \eta\left|\lambda_{k}\right|\right)}\left(\frac{z-\lambda_{n}}{3 \eta\left|\lambda_{n}\right|}\right)^{\mu_{n}} .
$$

We may remove the factor corresponding to $n=k$ thus obtaining the polynomial

$$
q_{\Lambda}^{k}(z, \eta)=q_{\Lambda}\left(z, \lambda_{k}, \eta\right)\left(\frac{z-\lambda_{k}}{3 \eta\left|\lambda_{k}\right|}\right)^{-\mu_{k}}=\prod_{\substack{\lambda_{n} \in B\left(\lambda_{k}, \eta\left|\lambda_{k}\right|\right) \\ n \neq k}}\left(\frac{z-\lambda_{n}}{3 \eta\left|\lambda_{n}\right|}\right)^{\mu_{n}}
$$

Evaluating at $\lambda_{k}$ gives

$$
q_{\Lambda}^{k}\left(\lambda_{k}, \eta\right)=\prod_{\substack{\lambda_{n} \in B\left(\lambda_{k}, \eta\left|\lambda_{k}\right|\right) \\ n \neq k}}\left(\frac{\lambda_{k}-\lambda_{n}}{3 \eta\left|\lambda_{n}\right|}\right)^{\mu_{n}} .
$$

We are now ready to define $S_{\Lambda}$. We let

$$
S_{\Lambda}:=\lim _{\eta \rightarrow 0} \liminf _{k \rightarrow \infty} \frac{\log \left|q_{\Lambda}^{k}\left(\lambda_{k}, \eta\right)\right|}{\left|\lambda_{k}\right|}
$$

Remark 1.1. Since $\left|q_{\Lambda}^{k}\left(\lambda_{k}, \eta\right)\right|<1$, the relation $S_{\Lambda} \leqslant 0$ is always true.

Theorem 1.1. Let $d$ be a non-negative real number and let $\xi$ be a real number. Then there exists a multiplicity sequence $\Lambda=\left\{\lambda_{n}, \mu_{n}\right\}_{n=1}^{\infty}$ satisfying (1.3), and a Taylor-Dirichlet series of the form

$$
\sum_{n=1}^{\infty}\left(\sum_{k=0}^{\mu_{n}-1} c_{n, k} z^{k}\right) e^{\lambda_{n} z}
$$

with

$$
\limsup _{n \rightarrow \infty} \frac{\log C_{n}}{\lambda_{n}}=\xi, \quad \text { where } \quad C_{n}=\max \left\{\left|c_{n, k}\right|: k=0,1, \ldots, \mu_{n}-1\right\},
$$

so that this series has no singularities on its abscissa of convergence $\operatorname{Re} z=-\xi$. 


\section{Proof of Theorem 1.1}

Suppose that a multiplicity-sequence $\Lambda=\left\{\lambda_{n}, \mu_{n}\right\}_{n=1}^{\infty}$ has positive real terms and the Valiron conditions (1.4) are satisfied. Suppose also that $S_{\Lambda}$ is negative. Let $\xi$ be a real number and denote by $C_{\xi}$ the class of all the double-indexed sequences of the form $\left\{c_{n, k}: n \in \mathbb{N}, k=\right.$ $\left.0,1, \ldots \mu_{n}-1\right\}$ so that

$$
\limsup _{n \rightarrow \infty} \frac{\log C_{n}}{\lambda_{n}}=\xi, \quad \text { where } \quad C_{n}=\max \left\{\left|c_{n, k}\right|: k=0,1, \ldots, \mu_{n}-1\right\} .
$$

For each element in the class $C_{\xi}$ consider the Taylor-Dirichlet series (1.2). Since the Valiron conditions are satisfied, then such a series is analytic in the half-plane $\operatorname{Re} z<-\xi$ having the line $\operatorname{Re} z=-\xi$ as the abscissa of convergence. Assuming all of the above, O.A. Krivosheeva proved [9, Thm. 3.1] that there exists a Taylor-Dirichlet series (1.2) having no singularities on the abscissa and continued analytically in a half-plane $\operatorname{Re} z<-\xi+\beta$ for some $\beta>0$.

Hence, in order to prove Theorem 1.1, it suffices to construct a multiplicity-sequence $\Lambda^{\prime \prime}$ with positive real terms, uniformly separated, having finite density $d \geqslant 0$ and $S_{\Lambda^{\prime \prime}}<0$. Our first step is to construct such a multiplicity-sequence $\Lambda$ with density zero. Our second step is to add a sequence $\Lambda^{\prime}$ having positive density $d$, with positive real simple terms which are uniformly separated. The union of $\Lambda$ with $\Lambda^{\prime}$ yields a new multiplicity-sequence $\Lambda^{\prime \prime}$ with the desired properties.

2.1. First step: an example of $\Lambda$ with density zero and $S_{\Lambda}<0$. For each positive integer $n$, consider the set with multiple terms

$$
\left\{n^{2} \cdot 10^{n}+k, 10^{n}\right\} \quad k=1,2, \ldots, n .
$$

That is:

for $n=1$ we have $\{11,10\}$, meaning that the number 11 is repeated 10 times,

for $n=2$ we have $\{401,100\}$ and $\{402,100\}$,

for $n=3$ we have $\{9001,1000\},\{9002,1000\}$ and $\{9003,1000\}$,

and so on. Consider then the infinite set

$$
\left\{n^{2} \cdot 10^{n}+k, 10^{n}\right\} \quad n \in \mathbb{N} \quad k=1,2, \ldots, n,
$$

and rewrite it as a multiplicity-sequence $\Lambda=\left\{\lambda_{n}, \mu_{n}\right\}_{n=1}^{\infty}$ such that

$$
\lambda_{1}=11, \quad \lambda_{2}=401, \quad \lambda_{3}=402, \quad \lambda_{4}=9001, \quad \lambda_{5}=9002, \quad \lambda_{6}=9003, \quad \ldots
$$

with respective multiplicities

$$
\mu_{1}=10, \quad \mu_{2}=100, \quad \mu_{3}=100, \quad \mu_{4}=1000, \quad \mu_{5}=1000, \quad \mu_{6}=1000, \quad \ldots
$$

We observe that for each $\lambda_{n}$ there is some $m \in \mathbb{N}$ and $i \in\{1,2, \ldots, m\}$ so that

$$
\lambda_{n}=m^{2} \cdot 10^{m}+i \text { and } \mu_{n}=10^{m} .
$$

Now, if $\lambda_{n}=m^{2} \cdot 10^{m}+1$ with $\mu_{n}=10^{m}$, then its previous term $\lambda_{n-1}$ will be $\lambda_{n-1}=$ $(m-1)^{2} \cdot 10^{m-1}+m-1$ with $\mu_{n-1}=10^{m-1}$. If however $\lambda_{n}=m^{2} \cdot 10^{m}+i$ for $i>1$, then its previous $i-1$ terms will be $\lambda_{n-k}=m^{2} \cdot 10^{m}+i-k$ for $k=1,2, \ldots, i-1$ with multiplicities $\mu_{n-k}=10^{m}$.

We now claim that the following three conditions hold:

(1) $\frac{\mu_{n}}{\lambda_{n}} \rightarrow 0$ as $n \rightarrow \infty$,

(2) $\Lambda$ has density zero,

(3) $S_{\Lambda}<0$. 
Condition (1) is obvious and we proceed with (2). For each $t>0$, there is a unique $n \in \mathbb{N}$ such that $\lambda_{n} \leqslant t$ but $\lambda_{n+1}>t$. Write $u_{t, n}=t-\lambda_{n}$. Then

$$
\begin{aligned}
\frac{n_{\Lambda}(t)}{t} & =\frac{\sum_{\lambda_{n} \leqslant t} \mu_{n}}{t}=\frac{\mu_{1}+\mu_{2}+\cdots+\mu_{n}}{\lambda_{n}+u_{t, n}} \\
& =\frac{10+2 \cdot 10^{2}+3 \cdot 10^{3}+\cdots+(m-1) \cdot 10^{m-1}+i \cdot 10^{m}}{m^{2} \cdot 10^{m}+i+u_{t, n}} .
\end{aligned}
$$

Thus

$$
\frac{n_{\Lambda}(t)}{t}<\frac{m}{m^{2} \cdot 10^{m}}\left(10+10^{2}+10^{3}+\cdots+10^{m}\right)=\frac{1}{m \cdot 10^{m}} \cdot \frac{10\left(10^{m}-1\right)}{9} .
$$

As $t \rightarrow \infty$, then $n$ and $m$ tend to infinity as well, hence, the right hand-side of this inequality converges to 0 , thus $\Lambda$ has zero density.

Next we show that condition (3) holds as well. From (1.6) we get

$$
\log \left|q_{\Lambda}^{k}\left(\lambda_{k}, \eta\right)\right|=\sum_{0<\left|\lambda_{k}-\lambda_{n}\right|<\eta\left|\lambda_{k}\right|} \mu_{n} \log \left|\frac{\lambda_{k}-\lambda_{n}}{3 \eta \lambda_{n}}\right| .
$$

As before, we write $\lambda_{k}=m^{2} \cdot 10^{m}+i$ for some $m \in \mathbb{N}$ and $i \in\{1,2, \ldots, m\}$. Observe that for $\eta<1 / 10$ the only $\lambda_{n}$ satisfying the inequality $0<\left|\lambda_{k}-\lambda_{n}\right|<\eta\left|\lambda_{k}\right|$ are the ones of the form $\lambda_{n}=m^{2} \cdot 10^{m}+j$ for $j \in\{1,2, \ldots, m\} \backslash\{i\}$ with multiplicity $\mu_{n}=10^{m}$. For these $\lambda_{n}$ we have

$$
\left|\frac{\lambda_{k}-\lambda_{n}}{3 \eta \lambda_{n}}\right|<\frac{m}{3 \eta \cdot m^{2} \cdot 10^{m}}=\frac{1}{3 \eta \cdot m \cdot 10^{m}}
$$

and

$$
\mu_{n} \log \left|\frac{\lambda_{k}-\lambda_{n}}{3 \eta \lambda_{n}}\right|<10^{m} \cdot \log \frac{1}{3 \eta \cdot m \cdot 10^{m}}
$$

Thus

$$
\sum_{0<\left|\lambda_{k}-\lambda_{n}\right|<\eta\left|\lambda_{k}\right|} \mu_{n} \log \left|\frac{\lambda_{k}-\lambda_{n}}{3 \eta \lambda_{n}}\right|<m \cdot 10^{m} \cdot \log \frac{1}{3 \eta \cdot m \cdot 10^{m}}
$$

Hence,

$$
\frac{\log \left|q_{\Lambda}^{k}\left(\lambda_{k}, \eta\right)\right|}{\lambda_{k}}<-\frac{m \cdot 10^{m} \cdot \log 3 \eta}{2 \cdot m^{2} \cdot 10^{m}}-\frac{m \cdot 10^{m} \cdot \log m}{2 \cdot m^{2} \cdot 10^{m}}-\frac{m \cdot 10^{m} \cdot \log 10^{m}}{2 \cdot m^{2} \cdot 10^{m}}
$$

Therefore,

$$
\frac{\log \left|q_{\Lambda}^{k}\left(\lambda_{k}, \eta\right)\right|}{\lambda_{k}}<-\frac{\log 3 \eta}{2 m}-\frac{\log m}{2 m}-\log \sqrt{10}
$$

As $k \rightarrow \infty$, we have $m \rightarrow \infty$ as well. Observe also that the right hand-side of this inequality converges to $-\log \sqrt{10}$ as $m \rightarrow \infty$. Therefore,

$$
\liminf _{k \rightarrow \infty} \frac{\log \left|q_{\Lambda}^{k}\left(\lambda_{k}, \eta\right)\right|}{\lambda_{k}} \leqslant-\log \sqrt{10}, \quad \forall \eta \in(0,1 / 10) .
$$

By the definition of $S_{\Lambda}$ in 1.7 we get $S_{\Lambda} \leqslant-\log \sqrt{10}$, thus $S_{\Lambda}$ is negative. 
2.2. Second step: adding a sequence $\Lambda^{\prime}$ with positive density. Consider now a sequence $\Lambda^{\prime}=\left\{\lambda_{n}^{\prime}\right\}_{n=1}^{\infty}$ with positive real terms, uniformly separated, having density $d>0$. For example, let $\lambda_{n}^{\prime}=n / d$ for all $n \in \mathbb{N}$. Let $\Lambda$ be the multiplicity-sequence of the previous subsection and let $\Lambda^{\prime \prime}=\left\{\lambda_{n}^{\prime \prime}, \mu_{n}^{\prime \prime}\right\}_{n=1}^{\infty}$ where

$$
\Lambda^{\prime \prime}=\Lambda \cup \Lambda^{\prime} .
$$

Then $\Lambda^{\prime \prime}$ has density equal to $d$ as well. One also deduces that $S_{\Lambda^{\prime \prime}} \leqslant \inf \left\{S_{\Lambda^{\prime}}, S_{\Lambda}\right\}$ therefore $S_{\Lambda^{\prime \prime}}<0$.

To finish the proof of Theorem 1.1 we have to make sure that $\Lambda^{\prime \prime}$ has uniformly separated terms. In order to achieve this, we can subtract any terms of $\Lambda^{\prime}$ that may lie in $\bigcup_{m=1}^{\infty} I_{m}$, where $I_{m}$ is the interval

$$
\left[m^{2} \cdot 10^{m}, m^{2} \cdot 10^{m}+m+1\right] .
$$

We now claim that this subset of $\Lambda^{\prime}$ removed has density zero. Indeed, assuming that $\lambda_{n+1}^{\prime}-\lambda_{n}^{\prime} \geqslant$ $\delta$ for some $\delta>0$ for all $n \in \mathbb{N}$, then $(m+2) / \delta$ is an upper bound for the number of terms of $\Lambda^{\prime}$ in the interval $I_{m}$. Hence $k^{2} / \delta$ is an upper bound for such terms in the finite union $\bigcup_{m=1}^{k} I_{m}$ and this is also an upper bound for the terms removed in the interval $\left[0, k^{2} \cdot 10^{k}+k+1\right]$. We then easily deduce that the set removed has density zero. Hence the remaining set, rename it as $\Lambda^{\prime}$, has density $d$.

Letting again $\Lambda^{\prime \prime}=\Lambda \cup \Lambda^{\prime}$, gives a multiplicity-sequence with density $d$, positive real terms and $S_{\Lambda^{\prime \prime}}<0$. Observe also that due to the removal of any possible terms of $\Lambda^{\prime}$ lying in $\bigcup_{m=1}^{\infty} I_{m}$, the terms of $\Lambda^{\prime \prime}$ will now be uniformly separated, thus achieving our goal. The proof of Theorem 1.1 is now finished.

\section{A. The Density COndition Implies (1.4)}

Observe that the density condition implies that $\sup _{n \in \mathbb{N}} n / \lambda_{n}<\infty$. Hence the first condition in (1.4) is valid since

$$
\frac{\log n}{\lambda_{n}}=\frac{\log n}{n} \cdot \frac{n}{\lambda_{n}} \rightarrow 0, \quad n \rightarrow \infty .
$$

Suppose now that the second condition in (1.4) does not hold. Then there is some $\tau>0$ and a subsequence $\left\{\lambda_{n_{k}}, \mu_{n_{k}}\right\}_{k=1}^{\infty}$ so that $\mu_{n_{k}} / \lambda_{n_{k}} \geqslant \tau$ for all $k \in \mathbb{N}$. Since $\Lambda$ has density $d$, then for every $\epsilon>0$ there is some $t(\epsilon)$ so that

$$
d-\epsilon \leqslant \frac{n_{\Lambda}(t)}{t} \leqslant d+\epsilon, \quad \forall t \geqslant t(\epsilon) .
$$

Taking $\epsilon=\tau / 10$, we have

$$
d-\frac{\tau}{10} \leqslant \frac{n_{\Lambda}(t)}{t} \leqslant d+\frac{\tau}{10}, \quad \forall t \geqslant t(\tau / 10) .
$$

Now, for each $t>0$, there is some $n \in \mathbb{N}$ such that $\lambda_{n} \leqslant t$ but $\lambda_{n+1}>t$. Let $u_{t, n}:=t-\lambda_{n}$ for $t \in\left[\lambda_{n}, \lambda_{n+1}\right)$. From this point on we assume that all $\lambda_{n}>t(\tau / 10)$. Hence we have

$$
d-\frac{\tau}{10} \leqslant \frac{\mu_{1}+\mu_{2}+\cdots+\mu_{n}}{\lambda_{n}+u_{t, n}} \leqslant d+\frac{\tau}{10}, \quad \forall t \in\left[\lambda_{n}, \lambda_{n+1}\right) .
$$

Thus,

$$
d-\frac{\tau}{10} \leqslant \frac{\mu_{1}+\mu_{2}+\cdots+\mu_{n_{k}}}{\lambda_{n_{k}}+u_{t, n_{k}}} \leqslant d+\frac{\tau}{10}, \quad \forall t \in\left[\lambda_{n_{k}}, \lambda_{n_{k}+1}\right) .
$$

where we assumed earlier that $\mu_{n_{k}} / \lambda_{n_{k}} \geqslant \tau$ for all $k \in \mathbb{N}$. Letting $t=\lambda_{n_{k}}$, thus $u_{t, n_{k}}=0$, gives

$$
d-\frac{\tau}{10} \leqslant \frac{\mu_{1}+\mu_{2}+\cdots+\mu_{n_{k}}}{\lambda_{n_{k}}} \leqslant d+\frac{\tau}{10} .
$$


Similarly we have

$$
d-\frac{\tau}{10} \leqslant \frac{\mu_{1}+\mu_{2}+\cdots+\mu_{n_{k}-1}}{\lambda_{n_{k}-1}+u_{t, n_{k}-1}} \leqslant d+\frac{\tau}{10}, \quad \forall t \in\left[\lambda_{n_{k}-1}, \lambda_{n_{k}}\right) .
$$

Observe now that $\lambda_{n_{k}-1}+u_{t, n_{k}-1} \rightarrow \lambda_{n_{k}}$ as $t \rightarrow \lambda_{n_{k}}^{-}$. Then we get

$$
d-\frac{\tau}{10} \leqslant \frac{\mu_{1}+\mu_{2}+\cdots+\mu_{n_{k}-1}}{\lambda_{n_{k}}} \leqslant d+\frac{\tau}{10} .
$$

Since $\mu_{n_{k}} / \lambda_{n_{k}} \geqslant \tau$ then

$$
\frac{\mu_{1}+\mu_{2}+\cdots+\mu_{n_{k}-1}+\mu_{n_{k}}}{\lambda_{n_{k}}} \geqslant d-\frac{\tau}{10}+\tau=d+\frac{9 \tau}{10} .
$$

But this contradicts the right hand-side of inequality (A.1), thus the second condition in (1.4) is indeed valid.

\section{BIBLIOGRAPHY}

1. P. Koosis. The logarithmic integral. II. Cambridge Studies in Advanced Mathematics. 21. Cambridge University Press, Cambridge (1992).

2. N. Arakelian, W. Luh, J. Müller. On the localization of singularities of Lacunar power series // Complex Var. and Ell. Eqns. 52:7, 561-573 (2007).

3. A. Eremenko. Densities in Fabry's theorem, // Illinois J. Math. 52:4, 1277-1290 (2008).

4. A. Eremenko. A version of Fabry's theorem for power series with regularly varying coefficients // Proc. Amer. Math. Soc. 136:12, 4389-4394 (2008).

5. R. P. Boas. Jr. Entire functions. Academic Press, New York (1954).

6. N. Levinson, Gap and density theorems. Amer. Math. Soc. Colloq. Publ. 26. Amer. Math. Soc., New York (1940).

7. V. Bernstein. Leçons sur les progrès récents de la théorie des séries de Dirichlet. Gauthier-Villars, Paris (1933).

8. S. Mandelbrojt. Dirichlet series. Principles and methods D. Reidel Publishing Co., Dordrecht (1972).

9. O. A. Krivosheeva., Singular points of the sum of a series of exponential monomials on the boundary of the convergence domain // Alg. Anal. 23:2, 162-205 (2011). [St. Petersburg Math. J. 23:2, 321$350(2012)]$.

10. M.G. Valiron, Sur les solutions des équations différentielles linéaires d'ordre infini et á coefficients constants // Ann. Ecole Norm. (3) 46, 25-53 (1929).

11. A.S. Krivosheev, A fundamental principle for invariant subspaces in convex domains // Izv. RAN. Ser. Mat. 68:2, 71-136 (2004). [Izv. Math. 68:2, 291-353 (2004).]

Elias Zikkos,

Department of Mathematics and Statistics,

POB 20537, University of Cyprus,

1678 Nicosia, Cyprus

E-mail: zik@ucy.ac.cy 